\title{
Randomized poly-encrypted image exploiting chaotic beheviour
}

\author{
Bouslehi Hamdi \\ University of Science \\ and Technology of Tunis, Tunisia \\ Email: hamdouchb@gmail.com
}

\author{
Seddik Hassen \\ University of Science \\ and Technology of Tunis, Tunisia \\ Email: seddikhassene@yahoo.fr
}

\author{
Amaria Wael \\ University of Science \\ and Technology of Tunis, Tunisia \\ Email: amaria.wael@ hotmail.fr
}

Received: April 5, 2021. Revised: Novemeber 18, 2021. Accepted: December 27, 2021. Published: January 13, 2022.

\begin{abstract}
The security of digital data has become an essential need. Because the storage, transmission of data social networking has became inevitable, the need to ensure that data is no longer a luxury but an absolute necessity. The picture takes a large part of the data and its presence every day more important. in this paper, we use a new technique encryption which is poly encryption, this technique is based on the Decomposition of the image in a random manner, and each block will be encrypted by an algorithm that used different algorithm ...
\end{abstract}

Keywords-poly encryption, permutation function, logistic function, multiple key

\section{INTRODUCTION}

With the rapid exchange of data electronically thus, it is important to protect the confidentiality of data unauthorized access. Security vulnerabilities that may affect the user privacy and reputation. Thus, data encryption is widely used to confirm the security in open networks such as the Internet. Because of the substantial increase in the transmission of digital data via the Internet, the security of digital images has become more prominent and attracted a lot of attention in the digital world today. In addition, the extension of multimedia technology in our the company has promoted digital images play a more important role role that traditional texts, which require serious protection the privacy of users for all applications. Each data type has its characteristics and therefore different techniques should be used to protect confidential image from unauthorized access. Most encryption algorithms available are used for text data. However, due to large data size and realtime requirements,algorithms that are suitable for text data can not be appropriate for multimedia data. Classical cryptographic algorithms such as RSA, DES, and ... are inefficient for image encryption due to the inherent characteristics of the image, particularly high the image data volume. Many researchers proposed different imageencryption schemes to overcome the problems of image encryption [1, 2, 3, and 4].

In this research, we tried to find one simple algorithms, fast and secure encryption image using a random permutation function and characteristics of chaotic functions by changing the intensity of pixels of an encoding using a logical operator and these techniques will be used in an image, but each algorithm will be used for encrypting a block of the original image decomposed into advance randomly, so this encryption technique colled poly encryption. According to large space in the main chaotic functions, this method is very robust.

Finally, this new encryption technique is very sensitive to small changes in key so even with the knowledge of approximate keys; it is not possible for the attacker to break the encryption.

\section{THE LOGISTIC FUNCTION}

The logistic map [5] is a polynomial mapping database, which a chaotic behavior, and may be obtained by a simple nonlinear dynamic equation [6]. Logistics is a recurrence for example, if the offense is not linear. This recurrence was popularized by the biologist Robert May in 1976. Its recurrence relation is.

$$
\tau\left(x_{n}\right)=x_{n+1}=\lambda x_{n}\left(1-x_{n}\right)
$$

The control parameter is fixed and chosen so that equation (1) has a chaotic behavior [7]. However, if we study the map with a different value of , it shows that it is a trigger for the chaos. Mathematically, the Logistic map is written with $\mathrm{x}$ is a number between 0 and 1, and represents the initial condition. $\lambda$ : is a positive number [8].

\section{ALGORITHM ENCRYPTION BASED ON ITERATION OF THE IMPROVED LOGISTIC MAP}

After the iteration of the function of "Logistic map" $\mathrm{N}$ times, we obtain $\mathrm{N}$ value $x_{n}$ between 0 and 1 . with $x_{0}$ : the initial value and $0<x_{0}<1$ and $\lambda$ : control parameter. In our encryption algorithm we take $x_{0}=0.4582$ and $\lambda=3.759889$, we obtain a chaotic signal and the value generated chaotic sweep the entire range of value between 0 and 1 . After 70 iterations, the signal from equation 1 is summarized in (figure 1). Indeed, the chaotic function Logistic Map has several properties, such as frequency and sensitivity to initial conditions (this is a characteristic of all chaotic systems: if we take a different value which is very close to then the values from the iteration change completely. If we take a different value which is very close to the values of $\lambda$ the iteration changes dramatically: this can be seen by a simulation tool Matlab, in particular by the value of these functions which are completely random. Although they are limited from a few bands, the iterative values never give the impression to converge even after an infinite number of iterations. The change of control parameters $(\lambda)$ and the initial condition $\left(x_{0}\right)$ by very close values in order to know the decryption algorithm always gives cryptograms so radically different that it is interesting to use the function in logistic encryption). 


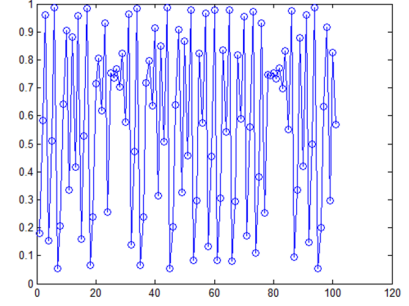

Fig. 1. Caption for this figure

\section{PROPOSED ENCRYPTION APPROACH}

This approach is based on four functions: a random number permutation function, a random function diagonal permutation , A feature that has changed is the intensity of the pixels image based on the encryption and a chaotic function "Logical function" function using the function also chaotic, all these algorithm will be used in an image, but each algorithm will be used to encrypt a block of the image decomposed in advance with a random manner.
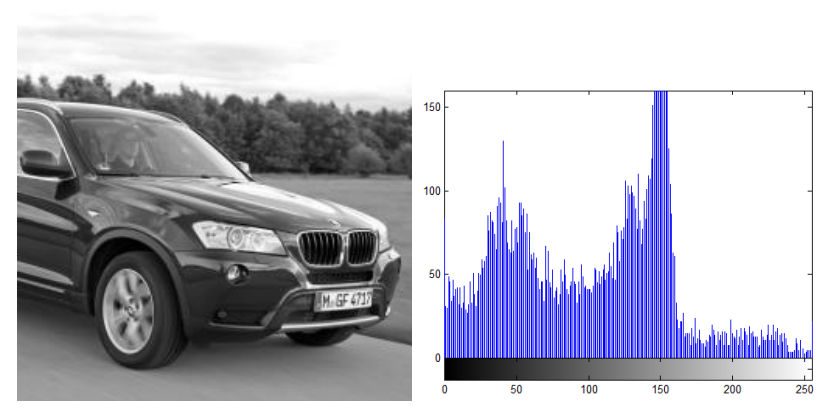

Fig. 2. Original image and its histogram

\section{A. First block}

The first block is encrypted with the permutation combined with the function of changing the intensity of the pixels.

1) Swap function: Mathematically our permutation function $P$ can be defined by the following equation: $\forall x \in N$ such as $P(x)=y$ so $\exists P^{-1}(x)$ with $P \circ P^{-1}(x)=I$ and $P^{-1}(y)-x=\varepsilon$ with $\varepsilon \longrightarrow 10^{-4}$.

The permutation has a paramount importance in this approach. It is used to make the system more safe and reliable. An image having pixels swapped does not make sense as opposed to the original image. So we take "I" the image to encrypt and $l$ is the permutation key that can be string or natural value. If our key is string we convert it to ASCII by using the flowing function:

ASCII: $X \longrightarrow Y$

As $\mathrm{X}$ : string

$\mathrm{Y}$ : vector of integers.

$l=A S C I I\left(l_{1}\right)$ with $l_{1} \in X$ and $Y \in l$ Our function contain $n$ round each used a key with $n$ is the length of our key. In each round the same function that is repeated but with a different key according to the key given thus explained the principle function we can detailed tow round which are as follows:

First round

$$
l=l_{1}, l_{2}, l_{3}, \ldots . . l_{n}
$$

With $l \in N^{*}$ and $i \in[1, n]$ and $n$ is the length of encryption key. The process of permutation is divided into several rounds. The round number is the length of the key "key1". So we start by the decomposition of our image into many vector whose its length is the value of $l_{1}$.

$$
I=C_{1}^{l_{1}}, C_{2}^{l_{1}}, \ldots \ldots \ldots C_{n}^{l_{1}}
$$

$I:$ is the image to encrypt.

$C_{1}^{l_{1}}$ : The first vector of image $I$ of length $l_{1}$.

After the decomposition of our image, we permute each bloc by using the permuted function.

$$
P\left(C_{1}^{l_{1}}=b_{1} b_{2} \ldots \ldots \ldots b_{l_{1}}\right)=C_{P 1}^{l_{1}}=b_{l_{1}} b_{l_{1}-1} \ldots \ldots \ldots b_{1}
$$

$C_{1}^{l_{1}}$ : The first vector from the image $I$ after permutation.

$$
P\left(C_{2}^{l_{1}}=b_{1} b_{2} \ldots \ldots \ldots b_{l_{1}}\right)=C_{P 2}^{l_{1}}=b_{l_{1}} b_{l_{1}-1} \ldots \ldots \ldots . . b_{2} b_{1}
$$

$C_{2}^{l_{1}}$ :The 2 nd vector from the image $I$ after permutation. Finely we have an image permutated by $l_{1}$ :

$$
P_{l_{1}}(I)=I_{\left(P, l_{1}\right)}=C_{P 1}^{l_{1}}, C_{P 2}^{l_{1}}, \ldots \ldots \ldots . . C_{P n}^{l_{1}}
$$

$I_{\left(P, l_{1}\right)}$ : Image after the first permutation.

\section{Second round}

$I_{\left(P, l_{1}\right)}$ : is the output of the first round and the input of the second round which start too by the decomposition of our image permuted by $l_{1}$ into many blocs whose its length is the value of $l_{2}$.

$$
I_{\left(P, l_{1}\right)}=C_{P 1}^{l_{1}}, C_{P 2}^{l_{1}}, \ldots \ldots \ldots C_{P n}^{l_{1}}=C_{1}^{l_{2}}, C_{2}^{l_{2}}, \ldots \ldots \ldots . . C_{n}^{l_{2}}
$$

As the first round after the decomposition of our image, we permute each vector by using the permuted function.

$$
\begin{gathered}
P\left(C_{1}^{l_{2}}=b_{1} b_{2} \ldots \ldots \ldots b_{l_{2}}\right)=C_{P 1}^{l_{2}}=b_{l_{2}} b_{l_{2}-1} \ldots \ldots . . b_{1} \\
P\left(C_{2}^{l_{2}}=b_{1} b_{2} \ldots \ldots \ldots b_{l_{2}}\right)=C_{P 2}^{l_{2}}=b_{l_{2}} b_{l_{2}-1} \ldots \ldots . . b_{1} \\
\cdot \\
\cdot \\
P\left(C_{n}^{l_{2}}=b_{1} b_{2} \ldots \ldots \ldots b_{l_{2}}\right)=C_{P n}^{l_{2}}=b_{l_{2}} b_{l_{2}-1} \ldots \ldots . . b_{1}
\end{gathered}
$$

Finally we have an image permutated by $l_{2}$ :

$$
P_{l_{2}}(I)=I_{\left(P, l_{2}\right)}=C_{P 1}^{l_{2}}, C_{P 2}^{l_{2}}, \ldots \ldots \ldots . . C_{P n}^{l_{2}}
$$

When we executed this function we have an image permuted by a random manner so there is not a mathematical model that ensures the passage from permuted image $I_{\left(P, l_{n}\right)}$ to original image $I$. Therefore, we can resume this permutation function by the following figure: 


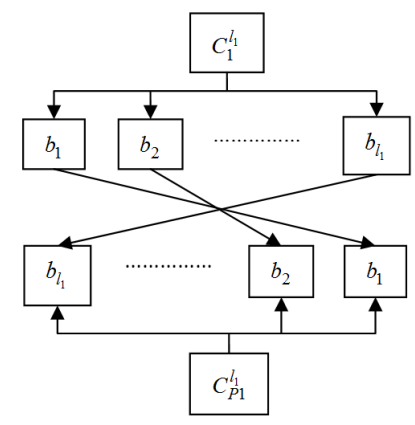

Fig. 3. Permutation function

2) Changing pixel intensity: After permutation performed on the message to be encrypted, which is in our case an image, we change the intensity to complicate the task. This procedure is to change the intensity of all image pixels by reducing or adding a random value between 0 and $d / 2$, generated by a logistic function $F$.

With $x_{0}=0.4582$ and $\lambda=3.759889$.

" $d$ " denotes the image dynamic, $d=256$ for the case of images coded on 8 bits.

$$
F=d_{1} d_{2} d_{3} \ldots \ldots \ldots d_{n}
$$

With $d_{1}$ : a binary value

$$
F=D_{1} D_{2} D_{3} \ldots \ldots \ldots . d_{n / 7}
$$

With $D_{1}=d_{1} d_{2} \ldots \ldots d_{7}$ : the first 7 binary values.

$I(i, j)$ Is the pixel coordinate $(i, j)$ with $i \in[1 . . h]$ and $j \in$ $[1 . . l]$ such that $h$ is the number of line and $l$ is the number of columns.

If $I(i, j)<d \backslash 2$ then:

$$
I_{c}(i, j)=I(i, j)+V_{K}
$$

else

$$
I_{c}(i, j)=I(i, j)-V_{K}
$$

$V_{K}$ : The value decimal of $D_{K}$ from the logistic function. else

$$
V_{K}=\sum_{i=1}^{n} 2^{i-(k(i-1))} * d_{i-(k(i-1))}
$$

$V_{K}$ is a random value belong to the interval $[0, \mathrm{~d} / 2]$.
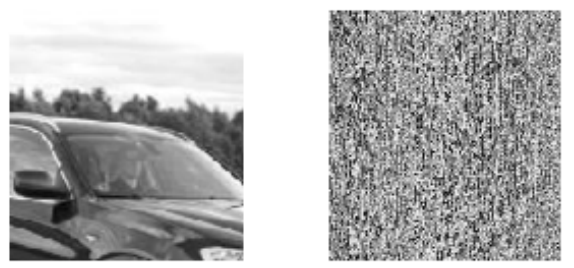

Fig. 4. Original block and the correspondent encrypted one

\section{B. Second block}

the second block will be encrypted with the xor function combined with Changing pixel intensity.
1) Encryption: For a more secure encryption system, we try to complicate the task. Each pixel intensity found after steps "1" and "2", which represent the permutation and change of intensity, is modified by the XOR function whose input pixel intensity change and a random value generated using a logistic function.

The XOR operator is extremely common as a component in more complex ciphers. By itself, using a constant repeating key, a simple XOR cipher can trivially be broken using frequency analysis. If the content of any message can be guessed or otherwise known then the key can be revealed. Its primary merit is that it is simple to implement, and that the XOR operation is computationally inexpensive. A simple repeating XOR cipher is therefore sometimes used for hiding information in cases where no particular security is required.

$$
F=d_{1} d_{2} d_{3} \ldots \ldots \ldots d_{n}
$$

Binarising the image:

$$
\begin{gathered}
I=I_{1} I_{2} I_{3} \ldots \ldots \ldots I_{n} \\
I_{c r y}=I \oplus F
\end{gathered}
$$

After this encryption method we used the function of changing pixels intensity to have finally the flowing encrypted image.
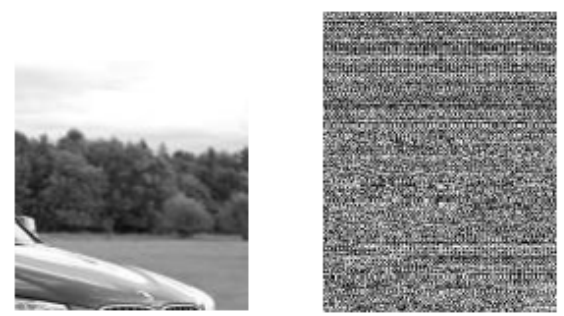

Fig. 5. Original block and the correspondent encrypted one

\section{Third block}

the third block will be the diagonal permutation function combined with Changing pixel intensity.

TABLE I. IMAGE MATRIX

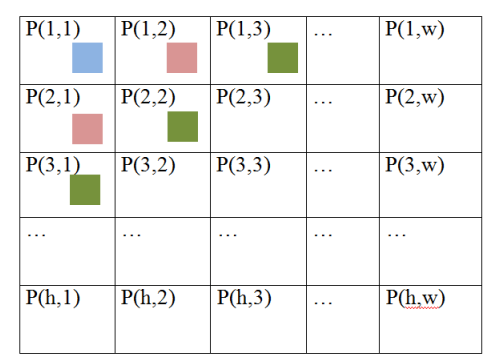

1) Diagonal permutation function: We set $P_{d}(M) \longrightarrow$ $M_{P}$ permutation function that will describe immediately. After encrypting the matrix corresponds to the image $M(i, j)$ which has $h$ lines and $w$ columns. We take each diagonal line of the resulting image. It performs translations circular, the number of translation and is randomly generated using a logistic function. For example: 
When $i=1$ we take only the first pixel.

When $i=2$ we take the permuted diagonal line that contains the two pixels $M(2,1)$ and $M(1,2)$ and the permuted with the following function:

$$
P_{s}^{n}\left(M_{2,1}, M_{1,2}\right)
$$

Such as $n$ : the number of permutations that will be made and $" s "$ is the permutation of its (right or left) A general manner permutation function is often written as follows: When $i=k$ we take the permuted diagonal line that contains the following pixels $(M(k, 1), M(k-1,2), \ldots \ldots \ldots . . M(1, k))$ so the permuted with the following function:

$$
P_{s}^{n}(M(k, 1), M(k-1,2), \ldots \ldots \ldots M(1, k))
$$
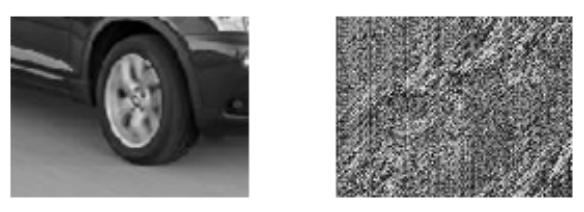

Fig. 6. Original block and the correspondent encrypted one

\section{Fourth block}

the fourth block will be encrypt by using the both permutation functions and Changing pixel intensity combined with the chaotic encryption.

1) encryption algorithm of the fourth block: to encrypt this block we choose to use the all algorithm that we used before in the same time.So the encryption algorithm of the fourth block can be present by the flowing diagram.

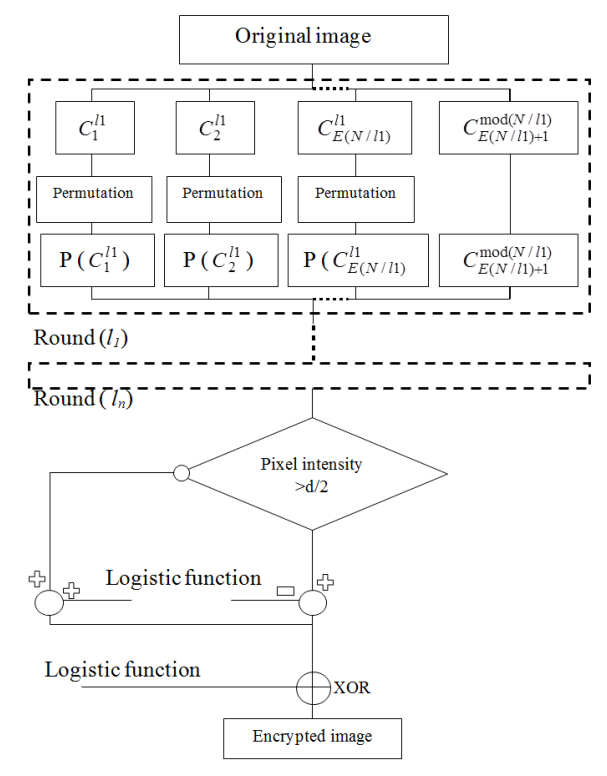

Fig. 7. Diagram of a new encryption approach

The folowing figure can show as the fourth block and its ecryption block correspondent.
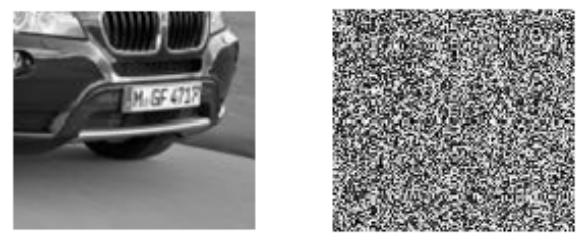

Fig. 8. Original block and the correspondent encrypted one

Now we can combine all these blocks in the same image.

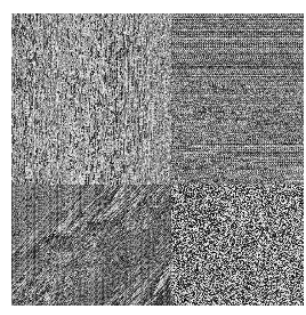

Fig. 9. Encrypted image

The security operation does not stop here we will also use a very simple technique may very efficient, we'll split the image into small blocks and Sev going to swapped a random manner as we present the following figure.
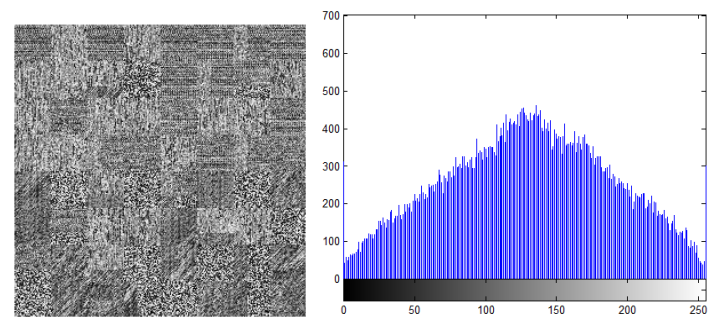

Fig. 10. Encrypted and permuted image and its histogram

\section{E. Image decryption steps}

1) Decryption function : Once the image is encrypted and transmitted just go the opposite way to recover the original image. The last procedure performed on the image during encryption is the XOR function, which has the original image as input whose pixels are switched and having intensities modified and logistics values. This procedure will be the first to perform during decryption by introducing the same parameters that are sent as "key".

$$
\begin{gathered}
I_{c r y}=I_{1} I_{2} I_{3} \ldots \ldots \ldots . . . I_{n} \\
I=I_{c r y} \oplus F
\end{gathered}
$$

2) Changing intensity: Original intensity of the image are modified, therefore they must be restored by adding (or subtracting) the same value subtracted (or added) during the process of changing intensity. As indicated previously the choice of the operator "+" or "-" is stored in a vector that is sent as a key. 
$i m-b$ : is a binary image formed during encryption process. If $i m-b=0$ then:

$$
I(i, j)=I_{c}(i, j)-V_{K}
$$

else

$$
I(i, j)=I_{c}(i, j)+V_{K}
$$

$V_{K}$ : The value decimal derived from the logistic function.

3) Permutation: The last operation to be done to recover the original image is permutation one more time.

$$
\begin{gathered}
P_{l_{n}}^{-1}\left(I_{\left(P, l_{n}\right)}\right)=P_{l_{n}}^{-1}\left(C_{P 1}^{l_{n}}, C_{P 2}^{l_{n}}, \ldots \ldots \ldots . . . C_{P n}^{l_{n}}\right)=I_{\left(P, l_{(n-1)}\right)}(26) \\
P_{l_{(n-1)}}^{-1}\left(I_{\left(P, l_{(n-1)}\right)}\right)=P_{l_{n}}^{-1}\left(C_{P 1}^{l_{(n-1)}}, C_{P 2}^{l_{(n-1)}}, \ldots \ldots \ldots . . . C_{P n}^{l_{(n-1)}}\right)=
\end{gathered}
$$

$$
I=C_{1}^{l_{1}}, C_{2}^{l_{1}}, \ldots \ldots \ldots \ldots C_{n}^{l_{1}}
$$

Once completed, the original image is covered. For a better interpretation of the results, we calculate the difference between the original image and the image decrypted. Losses for this approach is zero, no data is lost.
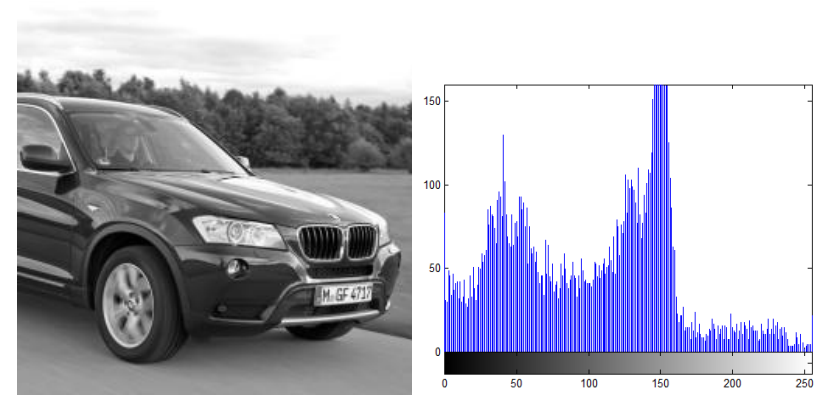

Fig. 11. Decrypted image

\section{RESULTS AND INTERPRETATION}

\section{A. Correlation between adjacent pixels}

For an ordinary image each pixel is highly correlated with its adjacent pixels in the horizontal or vertical direction. An encryption algorithm should produce images ideal encrypted with the correlation between adjacent pixels is negligible. The following figures show the correlation between the tested horizontally or vertically adjacent pixels of the image.
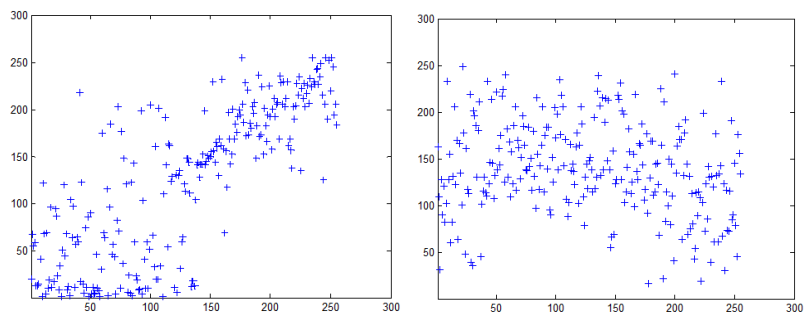

Fig. 12. Horizontal pixels correlation of our image before and after encryption
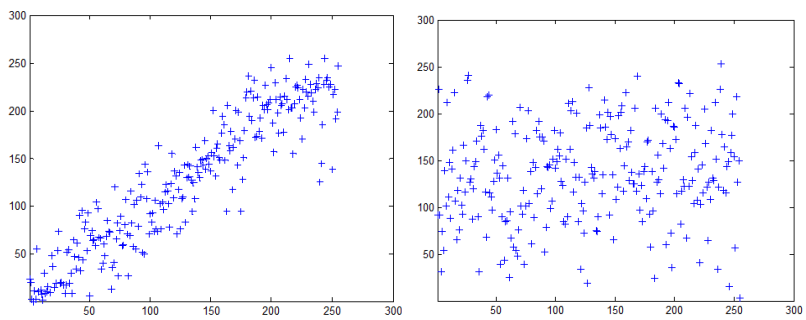

Fig. 13. Vertical pixels correlation of our image before and after encryption
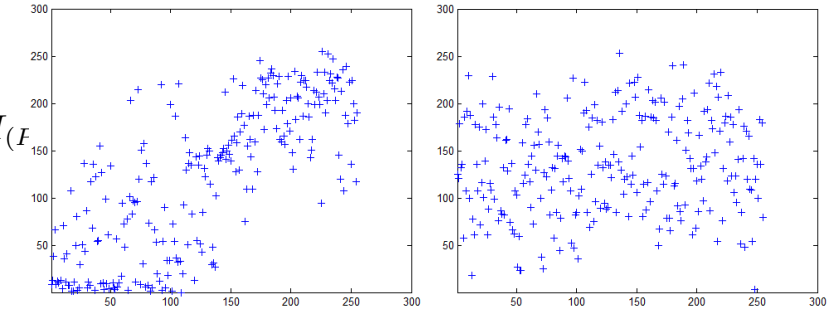

Fig. 14. Digonal pixels correlation of our image before and after encryption

It is clear that the correlation between adjacent pixels is dispersed in the image outputted by the encryption process. That means that the proposed algorithm is efficient because the pixels relation is broken and no relation can be established between them to identify the algorithm structure.

\section{B. mathematical tests}

Evaluation tools are used to evaluate the encryption performance so we must quantify its performance and characteristics. Among these tests, we include the mean square error (MSE), Peak Signal to Noise Ratio (PSNR), correlation and subtraction. The results of this case are presented in the table below. So it can be concluded from results obtained in this code that

TABLE II. RESULTS AND INTERPRETATION

\begin{tabular}{|l|l|l|}
\cline { 2 - 3 } \multicolumn{1}{l|}{} & Formula & Value \\
\hline MSE & $M S E=\frac{\sum_{i=1}^{n}\left(I_{i}-I^{*}\right)}{n}$ & 0 \\
\hline PSNR & $P S N R=10 \log _{10}\left(\frac{255^{2}}{M S S E}\right)$ & Inf \\
\hline Correlation & $\operatorname{Crr}\left(I, I^{*}\right)=\sum_{i=1}^{N} \sum_{j=1}^{M} \delta\left(I-I_{i}\right) \delta\left(I^{*}-I_{j}\right)$ & 1 \\
\hline Subtraction & $\operatorname{sub}=\sum_{i=1}^{n}\left(I_{i}-I_{i}^{*}\right)$ & 0 in each pixel \\
& & Image of Subtraction \\
\hline
\end{tabular}

we havent any loss of information. The decrypted image and the original one are the same which means that our algorithm is reversible.

\section{CONCLUSION}

In this paper we presented a new ecryption method that contain all the criterion of an encryption algorithm that is 
robust: the randomness of the function used and of decomposition of the image which is provided by the chaotic function and the function of the permutation, so that complexity of the key in our case it is ensured by the choice of key (value integer or character string), although the choice of encryption methods they have to be conservative and reversible, which is the case in our work.

\section{REFERENCES}

[1] M. Sharma and M.K. Kowar, Image Encryption Techniques Using Chaotic Schemes: a Review, International Journal of Engineering Science and Technology, vol. 2, no. 6, 2010, pp. 2359-2363.

[2] A. Jolfaei and A. Mirghadri, An Applied Imagery Encryption Algorithm Based on Shuffling and Bakers Map, Proceedings of the 2010 International Conference on Artificial Intelligence and Pattern Recognition (AIPR-10), Florida, USA, 2010, pp. 279-285.

[3] A. Jolfaei and A. Mirghadri, A Novel Image Encryption Scheme Using Pixel Shuffler and A5/1,Proceedings of The 2010 International Conference on Artificial Intelligence and Computational Intelligence (AICI10), Sanya, China, 2010.

[4] L. Xiangdong, Z. Junxing, Z. Jinhai, and H. Xiqin, Image Scrambling Algorithm Based on Chaos Theory and Sorting Transformation, IJCSNS International Journal of Computer Science and Network Security, vol. 8, no. 1, 2008, pp. 64-68.

[5] Luo, J.; Shi, H., Research of Chaos Encryption Algorithm Based on Logistic Mapping, IIH-MSP '06, International Conference on Intelligent Information Hiding and Multimedia Signal Processing, 2006, pp. 381383, Dec 2006.

[6] Rich Schlesinger, A Cryptography Cource for Non-Mathematicians, the 1st annual conference on information security curriculum development, K Kennesaw , Georgia, October 08-08,2004.

[7] Sudhir Keshari, Dr. S. G. Modani Image Encryption Algorithm based on Chaotic Map Lattice and Arnold cat map for Secure Transmission 1,2Department of Electronics and Communication Engineering, Malaviya National Institute of Technology, Jaipur, India. March 2011.

[8] Yong Wang, Xiaofeng Liao, Tao Xiang, Kwok-Wo Wong, Degang Yang Cryptanalysis and improvement on a block cryptosystem based on iteration a chaotic map Received 14 May 2006; accepted 6 November 2006 .Available online 20 November 2006,Communicated by A.R. Bishop.

\section{Creative Commons Attribution License 4.0 (Attribution 4.0 International, CC BY 4.0)}

This article is published under the terms of the Creative Commons Attribution License 4.0

https://creativecommons.org/licenses/by/4.0/deed.en US 